\title{
Descemet's membrane endothelial keratoplasty: surgical outcomes and endothelial cell count modelling from a UK centre
}

\author{
Mark A. P. Fajgenbaum ${ }^{1}$ - Nick Kopsachilis ${ }^{1}$ Emma J. Hollick ${ }^{1}$ \\ Received: 23 November 2017 / Revised: 7 May 2018 / Accepted: 21 May 2018 / Published online: 19 June 2018 \\ (c) The Royal College of Ophthalmologists 2018
}

\begin{abstract}
Purpose To report surgical outcomes and endothelial cell density (ECD) trends up to 2 years post-operatively in the first cohort of eyes undergoing Descemet's membrane endothelial keratoplasty (DMEK) for low-to-moderate risk indications at our UK centre. Tight corneal sutures were used to ensure high intraoperative pressure from intracameral air for 10-min and to maintain a good post-operative air fill. Outcomes were compared to a prior series at our unit of similar eyes having Descemet's stripping endothelial keratoplasty (DSEK).

Methods Retrospective, interventional series. Inclusions: all DMEKs with a minimum of 1-month follow-up. Exclusions: prior glaucoma surgery, penetrating keratoplasty or anterior chamber lens.

Results 88 DMEKs met inclusion. Median follow-up: 332 days. Main indication: Fuchs dystrophy (90\%). Complications: re-bubbling $2 \%$, primary failure $6 \%$, retinal detachment $2 \%$, rejection $3 \%$. At 3 months, BCVA $\geq 6 / 6$ and $\geq 6 / 9$ were $67 \%$ and $86 \%$, respectively. Graft survival at both 1 and 2 years was $94 \pm 2 \%$. Mean $( \pm$ SD) cell-loss at 1 month, 3 months, 6 months, 12 months and 24 months: $30 \pm 15 \%, 29 \pm 16 \%, 33 \pm 17 \%, 35 \pm 17 \%$ and $48 \pm 16 \%$, respectively. Compared to a prior series at our unit of 210 low-to-moderate risk DSEKs, cell loss was lower for DMEK at all time-points $(p<0.001)$ through to 12 months but was not statistically different at 24 months. Mixed-modelling of ECD over time demonstrated the convergence of DMEK and DSEK curves by 2 years.

Conclusions DMEK using tissue from UK eye-banks resulted in high survival and excellent visual acuity. ECD through 1 year was superior to DSEK in a similar cohort. Our low re-bubble rate (2\%) may be attributable to use of intracameral air at temporarily elevated intraocular pressure.
\end{abstract}

\section{Introduction}

Descemet's membrane endothelial keratoplasty (DMEK) was first described by Melles et al. in 2002 [1-3] and is now widely regarded as the preferred technique for the surgical management of corneal endothelial dysfunction, particularly for low-risk indications such as Fuchs' endothelial dystrophy (FED) [4]. In DMEK surgery, the diseased endothelium and its associated Descemet's membrane (DM) are replaced with corresponding layers from a healthy donor cornea. Unlike its closely related predecessor Descemet's stripping endothelial keratoplasty (DSEK), in DMEK the transplanted DM and endothelium are not accompanied by a

Mark A. P. Fajgenbaum

markfajgenbaum@gmail.com

King's College Hospital, London, UK lamella of donor stroma. DMEK therefore results in better preservation of the corneal architecture compared to DSEK, resulting in superior visual outcomes $[4,5]$.

Although widely adopted, the long-term survival of DMEK grafts has yet to be established due to the relative novelty of the technique. Even DSEK, which preceded DMEK in clinical practice by a few years [6], has little published data on graft survival rates beyond the first 5 years. In the absence of long-term data, trends in endothelial cell density (ECD) over time can provide useful insights into the future viability of transplanted tissue [7].

We have previously published data on ECD trends over time from our institution post-DSEK, representing the largest such series from the UK [8]. While our 5-year DSEK graft survival compared favourably to published literature, we found a higher peri-operative cell loss and correspondingly lower mean ECD counts across the first 5 years of follow-up compared to centres in the USA $[9,10]$ and Singapore [11]. The cause of this discrepancy is uncertain. 
We have previously noted that there is geographic variability in donor tissue characteristics such as donor age and cell counts, as well as in storage techniques used by eye banks, which may play a role in the resilience of endothelial cells during and after surgery [12].

In 2015, we changed the standard practice in our unit to performing DMEK rather than DSEK for most cases of endothelial dysfunction in keeping with international trends and best available evidence. In this report, we publish our 2year outcomes in DMEK surgery, with particular emphasis on ECD trends over time by comparison to our previously published data on DSEK.

\section{Methods}

This study was designed as a single-institution, retrospective, consecutive interventional series. It was approved by our institutional review board and adhered to the tenets of the Declaration of Helsinki. All patients provided informed consent prior to surgery.

\section{Recruitment and inclusion}

All DMEK grafts performed at our institution have been prospectively recorded on an anonymised corneal graft research database. The database collects pre-operative donor and recipient characteristics, intra-operative details, and post-operative data from each follow-up visit, including best corrected visual acuity (BCVA) via refraction or pinhole, intra-ocular pressure (IOP), graft viability, ECD and complications. In this study, data was extracted for analysis on all DMEK operations performed at our institution with a minimum of 4 weeks of follow-up, unless the graft had failed before such time. Grafts were excluded from the study if there were high-risk characteristics defined as iridocorneal endothelial (ICE) syndrome, previous penetrating keratoplasty (PK), previous glaucoma drainage surgery or presence of an anterior chamber lens implant.

\section{Study outcomes}

The main outcomes of the study were to determine the following:

(i) The incidence of post-operative complications, specifically the rates of partial graft detachment, rebubbling, graft rejection, raised IOP and graft failure.

(ii) Cumulative graft survival at 1 year and 2 years by Kaplan-Meier analysis.

(iii) Mean ECD for the cohort at 1 month, 3 months, 6 months, 12 months and 24 months; with a comparison to previously published counts from our own institution post-DSEK surgery, where similar indications were studied [8].

(iv) The trend in ECD over time by mixed non-linear regression modelling of an exponential decay function in an analysis similar to that published by our own institution for routine DSEK grafts [8] and by Riddlesworth et al. for PK data [13] from the Corneal Donor Study.

\section{Surgical technique}

\section{Donor preparation}

Donor preparation was performed immediately before transplantation using the SCUBA technique [14]. After staining the endothelium with $0.06 \%$ trypan blue (Visionblue $^{\circledast}$, DORC, Exeter, NH) the DM was scored with a reverse Sinskey's hook (Duckworth and Kent, Baldock, UK). The edge was grasped with a DMEK forceps (Geuder AG, Heidelberg, Germany) and carefully detached from the stroma in four quadrants leaving a small area of attachment centrally. The graft was punched with an $8.00-8.75 \mathrm{~mm}$ Barron corneal donor punch (Katena Products, Denville, $\mathrm{NJ}$ ), with the trephine size determined by the recipient cornea size. The final area of attachment was then peeled.

\section{Recipient}

All patients had an Nd:YAG laser iridotomy prior to surgery to reduce the likelihood of pupillary block. Surgery was performed under peribulbar or general anaesthesia depending on patient suitability. In combined cases, phacoemulsification was performed initially and a foldable acrylic IOL (Tecnis PCB100, AMO, Santa Ana, CA) inserted in the bag after which all viscoelastic was removed. An anterior chamber maintainer was then inserted. The patient's DM was removed with a reverse Sinskey's hook (Duckworth and Kent, Baldock, UK) to a diameter $0.25 \mathrm{~mm}$ greater than the size of the donor, using an ink mark on the cornea as a guide. Acetylcholine $1 \%$ (Miochol $^{\circledR}$, Novartis, Camberley, UK) was instilled intracamerally to miose the pupil. The donor DM scroll was stained again with $0.15 \%$ trypan blue (Membrane Blue ${ }^{\circledR}$, DORC, Exeter, NH) and injected into the patient's eye using a Geuder injector (Geuder AG, Heidelberg, Germany) on a $1 \mathrm{~mL}$ syringe. A higher concentration of trypan blue was chosen at this stage to improve the retention of stain. The graft was positioned centrally by repeatedly tapping on the surface of the cornea in a soft eye, with the endothelium down as previously described [15]. Air was injected underneath the graft. All corneal incisions were tightly sutured with 10/0 Nylon. The AC was completely filled with air to a higher than 
physiological IOP and this was left in place for $10 \mathrm{~min}$. Some air was then released in order to achieve physiological IOP.

\section{Follow-up protocol}

Grafts were reviewed at $2 \mathrm{~h}, 1$ week, 1 month, 3 months, 6 months, 12 months and annually thereafter, with additional visits arranged as clinically indicated. Corneal sutures were removed at the 1-month follow-up. From 1 month onwards, ECD was measured at each routine visit using a Confoscan 4 machine (Nidek Technologies Inc., Fremont, CA). For cell counts, the best image of the endothelium was chosen from multiple images of the central cornea and the machine's automated counting mode was used, with manual correction for any double counted cells. Our typical post-operative steroid regime comprised dexamethasone $0.1 \%$ drops reducing as follows: hourly for 2 days, 2 hourly for 5 days, 6 times a day for 1 week, 4 times a day for 3 months, 3 times a day for 2 months, 2 times a day for 2 months, once a day for 6 months, then alternate days lifelong.

\section{Statistics}

All statistical analyses were performed using SPSS version 20 (IBM, Armonk, NY). Numerical variables were described as mean \pm SD for normally distributed data and as median \pm range for non-normally distributed samples. Normality was confirmed by Shapiro-Wilk testing. 1-year and 2-year DMEK survival was calculated by Kaplan-Meier analysis. At each follow-up interval, mean ECD for DMEK was compared against mean ECD for prior data on DSEK using the Students' $t$-test. Homogeneity of variance was confirmed at each interval by Levene's testing. Mixed nonlinear regression modelling of DMEK cell counts was performed using an exponential decay function of the form: $\mathrm{ECD}=\mathrm{a} \cdot \mathrm{e}^{\mathrm{bt}}$, where ' $\mathrm{a}$ ' and ' $\mathrm{b}$ ' are constants and ' $\mathrm{t}$ ' is time in an analysis similar to that published by our own institution for routine DSEK grafts [8] and by Riddlesworth et al. for PK data [13] from the Corneal Donor Study. The mixed-model took account of both fixed-effects (time) and random effects (graft-related, time, intercept) with estimation by the maximum likelihood (ML) method and using unstructured variance-covariance, similar to our prior statistical work on DSEK.

\section{Results}

\section{Demographics and pre-operative details}

93 DMEK operations were performed at our institution over the study period. Four grafts were subsequently excluded because of previous PK surgery in the operated eye, while one graft was excluded because of less than 1 month of follow-up data. 88 DMEK operations on 87 eyes of 64 patients were included in the ongoing analysis. Of those included, most operations were undertaken in Caucasian patients $(91 \%, n=80)$ and in females $(60 \%, n=53)$. Mean patient age was $70 \pm 11$ years (range: $39-93$ years). The most common indication for surgery was FED $(90 \%, n=$ 79) while other indications included pseudophakic bullous keratopathy ( $[\mathrm{PBK}], 2 \%, n=2)$, herpes virus endotheliitis $(2 \%, n=2)$ and other causes $(5 \%, n=5) .95 \%(n=84)$ of operations were performed in eyes with no prior corneal graft surgery, while four eyes were repeat endothelial grafts: three with prior DSEK and one with prior DMEK. 94\% of eyes $(n=83)$ had no ocular co-morbidities apart from corneal endothelial disease with or without cataract. There were two cases of medically managed POAG, one case with epiretinal membrane and two cases with prior retinal detachment repair.

\section{Operative data and donor characteristics}

The majority of cases $83 / 88$ were performed directly by or under the supervision of a single consultant (EJH) but two other consultants from our unit who were also on the DMEK learning curve performed five cases. $73 \%$ of operations $(n=64)$ were combined phaco-DMEK while the remainder $(27 \%, n=24)$ were DMEK-only. Graft insertion was performed with the Geuder $2.4 \mathrm{~mm}$ injector in 80 cases (89\%) and the Geuder $3.2 \mathrm{~mm}$ injector in the remaining eight cases $(11 \%)$. Mean donor age was $66 \pm 11$ years (range: 34-85 years) with a mean donor count of $2660 \pm$ 215 cells $/ \mathrm{mm}^{2}$ (range: $2300-3390$ cells $/ \mathrm{mm}^{2}$ ). Follow-up ranged from 4 weeks to 30 months, with a median follow-up of 331 days.

\section{Post-operative events and complications}

Partial graft detachment was observed early in $34 \%(n=30)$ of cases. However, re-bubbling was only performed if more than $1 / 3$ of the graft was detached and was only required in $2 \%$ of grafts $(n=2)$. In the 28 cases where partial detachment involved less than $1 / 3$ of the graft area, the partial detachments all settled without rebubbling over the first month with a resolution of oedema. In some of these cases, there were minor folds at the edge of the graft but these were not visually significant. The release of intra-cameral air at the slit lamp was performed at $2 \mathrm{~h}$ in three grafts due to raised IOP. In total, $50 \%$ of eyes had a measurable IOP over $21 \mathrm{mmHg}$ at some point during the course of followup. This was managed conservatively or with topical medication and no grafts required surgical glaucoma input. Graft rejection was diagnosed in $3 \%$ of eyes $(n=3)$. In all 
Table 1 Mean ECD for DMEK compared to prior published outcomes from our institution for DSEK

\begin{tabular}{|c|c|c|c|c|c|c|c|}
\hline \multirow[t]{2}{*}{ Post-op interval } & \multicolumn{3}{|c|}{ DMEK } & \multicolumn{3}{|l|}{ DSEK $^{\mathrm{b}}$} & \multirow[t]{2}{*}{$p$-Value } \\
\hline & $N^{\mathrm{a}}$ & $\mathrm{ECD}$ cells $/ \mathrm{mm}^{2}($ mean $\pm \mathrm{SD})$ & $\%$ ECL & $N^{\mathrm{a}}$ & $\mathrm{ECD}$ cells $/ \mathrm{mm}^{2}($ mean $\pm \mathrm{SD})$ & $\%$ ECL & \\
\hline 1 month & $64 / 88$ & $1860 \pm 456$ & $30 \pm 15 \%$ & NA & NA & NA & - \\
\hline 3 months & $52 / 77$ & $1898 \pm 455$ & $29 \pm 16 \%$ & $140 / 210$ & $1570 \pm 370$ & $38 \pm 14 \%$ & $<0.001$ \\
\hline 6 months & $58 / 65$ & $1774 \pm 473$ & $33 \pm 17 \%$ & $164 / 205$ & $1510 \pm 390$ & $41 \pm 14 \%$ & $<0.001$ \\
\hline 12 months & $45 / 47$ & $1729 \pm 475$ & $35 \pm 17 \%$ & $153 / 185$ & $1440 \pm 420$ & $44 \pm 16 \%$ & $<0.001$ \\
\hline 24 months & $11 / 14$ & $1358 \pm 438$ & $48 \pm 16 \%$ & $126 / 155$ & $1340 \pm 420$ & $48 \pm 16 \%$ & 0.92 \\
\hline
\end{tabular}

$D M E K$ Descemet's membrane endothelial keratoplasty, $D S E K$ Descemet's stripping endothelial keratoplasty, $E C D$ endothelial cell density (mean $\pm \mathrm{SE}), \% E C L$ percentage endothelial cell loss, $N A$ not available

${ }^{a} N$ represents the number of grafts with documented ECD counts at that visit/the number of grafts reviewed at that visit

${ }^{\mathrm{b}}$ This data emanates from a prior published report from our institution on DSEK outcomes in routine indications such as FED and PBK [9]

'Student's $t$-test of mean ECD counts (DMEK vs DSEK)

cases, rejection was diagnosed within the first month due to an increase in corneal thickness which resolved following intensive hourly dexamethasone $0.1 \%$ drops administered for 1-2 weeks. Other documented events during follow-up were as follows: ocular surface issues, $16 \%(n=14)$; retinal detachment $(\mathrm{RD}), 2 \%(n=2)$; $\mathrm{CMO}, 2 \%(n=2)$; posterior capsule opacification (PCO), 2\% $(n=2)$; and iritis, $1 \%(n$ $=1$ ). The RD cases both presented at 2 months postoperatively and they were both successfully repaired with one vitrectomy procedure and maintained viable grafts throughout follow-up with vision preserved at $6 / 6$.

\section{Visual outcomes and graft survival}

The proportion of eyes attaining BCVA targets at the 3 months follow-up visit were as follows: $67 \%$ (48/72) achieved $\geq 6 / 6 ; 86 \%$ (62/72) achieved $\geq 6 / 9$ and $88 \%$ (63/72) achieved $\geq 6 / 12$. There were 16 viable grafts with no BCVA recorded at 3 months, either because the visit did not occur or the data was not inputted; but this missing data did not appear biased towards poorly performing grafts as $15 / 16$ (94\%) achieved $\geq 6 / 12$ at either the visit preceding (1 month) or following (6 months) the 3 months check. Primary graft failure occurred in five eyes $(6 \%)$ during the course of follow-up and was diagnosed within the first 14 days of surgery in all cases. There were no cases of late endothelial failure in this series. Cumulative graft survival ( \pm standard error) by Kaplan-Meier analysis was $94 \%( \pm 2 \%)$ at both year 1 and year 2 .

\section{Endothelial cell density (ECD) trends}

Mean ECD counts during follow-up are shown in Table 1 and a best-fit mixed-model of ECD over time for DMEK is shown in Fig. 1 by comparison to previous trends for DSEK data in similar patients from our own centre [8] and PK data from Riddlesworth et al. [13]. The DMEK regression line is

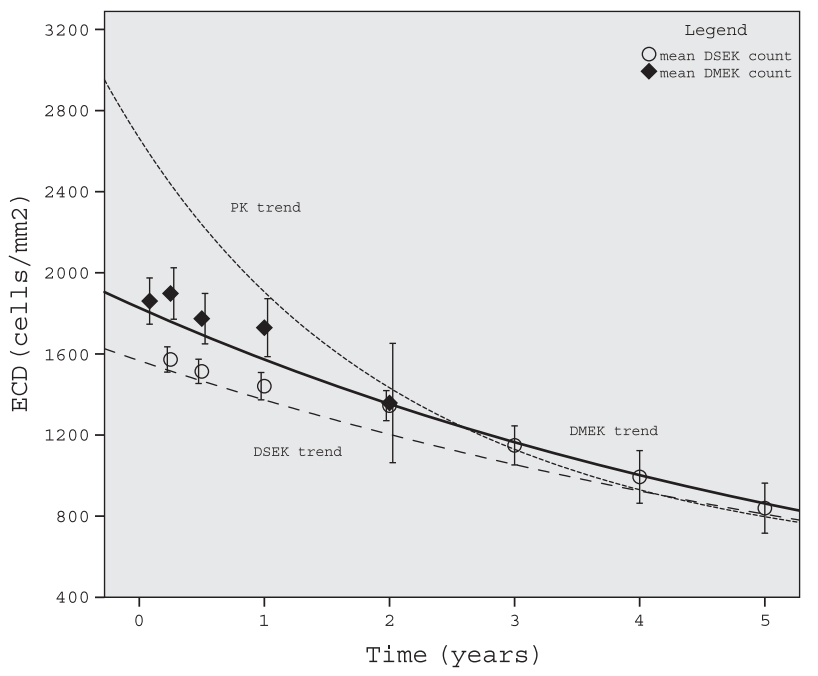

Fig. 1 Mixed non-linear regression models of ECD over time for (i) DMEK data from this series (solid line; equation: $E C D=1827 . \mathrm{e}^{-0.150 t}$ ); (ii) DSEK data previously published by our institution [9] (coarse dashed line; equation: $\mathrm{ECD}=1567 \cdot \mathrm{e}^{-0.132 \mathrm{t}}$ ) and (ii) $\mathrm{PK}$ data modelled by Riddlesworth et al. [13]. The black diamond markers represent the mean ECD for DMEK at 1 month, 3 months, 6 months, 1 year and 2 years; while the circular markers represent the mean ECD for DSEK at 3 months, 6 months, 1 year, 2 years, 3 years, 4 years and 5 years. The error bars represent the $95 \%$ confidence intervals of the means. It should be noted that the DSEK and DMEK regressions are derived from post-operative data-points only; such that the $y$-intercepts of these curves represent an estimation of the first post-operative cell counts at time-zero or in other words an estimation of cell loss associated with surgery. On the other hand, the regression performed by Riddlesworth for PK data utilised pre-operative donor cell counts in the regression, so that the $y$-intercept represents a pre-operative donor cell count value

a trend of post-operative cell counts only (pre-operative donor counts are not included as data points in the regression). As such, the $y$-intercepts provide an estimate of the cell count at time zero post-operatively $\left(1827\right.$ cells $/ \mathrm{mm}^{2}$ for DMEK), equivalent to a cell loss attributable to DMEK surgery of $31 \%$. 


\section{Discussion}

Our series represents the largest to date from a UK centre on DMEK surgical outcomes. Visual acuity results were excellent with $67 \%$ of eyes attaining a BCVA of $6 / 6$ or better and $86 \%$ attaining $6 / 9$ or better at 3 months. This compares favourably to BCVA outcomes from large multicentre [5] and single-centre [16-18] DMEK series emanating from international centres.

Re-bubbling was required in only $2 \%$ of cases in our series, which is among the lowest in the reported literature. Our re-bubble rate compares favourably with the low rates encountered in experienced, high-volume centres, such as that reported by Rodriguez-Calvo-De-Mora et al. in the Netherlands (3\%) [16].

Re-bubble rates with DMEK have varied widely across the literature with some groups reporting rates as high as 76-82\% [19, 20]. A recent, comprehensive literature review found a mean re-bubble rate of $29 \%$ for DMEK [4]. Graft detachment rates [5] and re-bubble rates [21] are known to improve with surgical experience. Our initial 'learning curve' DMEK cases were included in this series, but it is notable that we had 10 years of prior experience on over 300 DSEKs before transitioning to DMEK, which may have helped our technique and outcomes. We tightly suture all DMEK corneal wounds to ensure a high intraoperative IOP for $10 \mathrm{~min}$ and to maintain a good postoperative air-fill. We believe this manoeuvre helps graft attachment. Compared to our previously published DSEK outcomes [8], the re-bubble rate for our DMEKs was lower ( $2 \%$ vs $10 \%)$. This is in contrast to the findings of both a retrospective comparative series by Hamzaoglu et al. [17] and a systematic review by Singh et al. [22] where DMEK had higher overall re-bubble rates than DSEK [17, 22].

Primary graft failure occurred in $6 \%$ of cases in our DMEK series, which is similar to the rate found in the largest DMEK series to date by Oellerich et al. (multicentre, $7 \%$ ), although some high-volume units have reported primary failure rates as low as $0.2 \%$ [16].

Regression modelling of ECD over time (Fig. 1) for DMEK vs our prior series on DSEK showed that cell-loss attributable to DMEK surgery ( $y$-intercept of regression curve) was $31 \%$ compared to $39 \%$ for DSEK. The lower operative cell loss with DMEK contributed to significantly higher mean ECD values for DMEK grafts compared to DSEK grafts at our institution at 3 months, 6 months and 1 year. This is in contrast to the findings of Singh et al. who observed no difference in ECD counts between DSEK and DMEK at 6 months by meta-analysis across six comparative studies [22].

The discrepancy in post-operative cell counts at our institution between DSEK and DMEK surgery appears to have arisen principally from a higher-than-expected cell loss with DSEK. Our 6 months (41\%) and 12 months (44\%) cell loss rates with DSEK [8] were higher than other longitudinal DSEK series, such as those by Wacker et al. [10] (32\% at 12 months) and Price et al. [9] (37\% at 1 year) from the USA and by Ang et al. [11] (25\% at 12 months) from Singapore. On the other hand, our DMEK cell counts in this report compared favourably to the published DMEK literature. Our 6 months DMEK cell loss (33\%) was similar, if not superior to, reports emanating from units in the USA [17, 18], the Netherlands [16] and across international centres [5], where 6 months cell loss for DMEK has ranged between 28 and $40 \%$.

The cause of our unit's higher-than-expected cell loss with DSEK is uncertain. The majority of our DSEK grafts were inserted with either the Tan endoglide [69\%] (Angiotech, Vancouver, Canada) or the Busin glide [22\%] (Moria, USA) [8], which are widely used and are reported to have acceptable cell loss profiles [23, 24]. We have previously speculated about the potential role of donor age and cell counts [12]. It is notable that donor counts in our DSEK series were significantly lower (mean: 2550 cells $/ \mathrm{mm}^{2}$ ) than the aforementioned DSEK series' by Wacker et al. (mean 2925 cells $/ \mathrm{mm}^{2}$ ), Price et al. (3030 cells $/ \mathrm{mm}^{2}$ ) and Ang et al. $\left(2853\right.$ cells $\left./ \mathrm{mm}^{2}\right)$. On the other hand, our DMEK donor counts in this current report (mean 2660 cells $/ \mathrm{mm}^{2}$ ) have been more closely aligned to recent DMEK reports from the USA and elsewhere [5, 16-18]. The difficulty of young donor tissue tearing during preparation and creating tight, intra-operative DMEK scrolls (which is not an issue for DSEK) may be leading surgeons to favour comparably older tissue in countries that traditionally had access to young donor material for DSEK. This may be contributing to less discrepancy between donor material across countries and correspondingly more similar post-operative DMEK counts.

We have previously noted that cell loss after DSEK continues at a relatively constant rate from 3 months through to at least 5 years, with no discernible plateauing [8]. A similar phenomenon was observed in our DMEK series with a consistent cell loss through to 2 years. The mixed-model regressions of DSEK and DMEK tended towards subtle convergence over time (Fig. 1) with similar projected cell counts from about 2 years onwards. This suggests that endothelial cell survival and ultimately long-term graft survival may be similar for the two techniques; although larger datasets and longer follow-up are desirable to validate these trends. As yet there are few reports in the literature on DMEK outcomes beyond 4 years, but those that do exist have shown cell loss between 38 and 53\% [25-27], which appears comparable to DSEK 5-year cell loss of between 49 and $67 \%$ across studies $[8-11]$. 


\section{Conclusions}

DMEK surgery in our series, utilising tissue sourced from the UK eye banks, produced excellent visual acuity outcomes, high graft survival to 2 years and peri-operative cell loss on par with DMEK studies from international centres. The re-bubble rate in our DMEK series was one of the lowest yet reported at $2 \%$ and suggests that our technique of tightly suturing corneal wounds to ensure high intraoperative pressure from intracameral air for $10-\mathrm{min}$ and to maintain a good post-operative air fill may aid graft attachment. Longitudinal mixed-modelling of ECD over time for DMEK vs DSEK found a tendency towards model convergence by 2 years post-surgery, suggesting long-term graft survival between the two techniques may be closely comparable. We recommend further research to validate these trends to better assess long-term DMEK graft viability.

\section{Summary}

\section{What was known before}

- Published data from the UK transplant registry and our own UK centre have suggested that DSEK graft survival and endothelial cell counts during followup, respectively, may be inferior to published data from single centres in the USA and Singapore. There has been little data on DMEK graft survival and cell counts from the UK.

\section{What this study adds}

- DMEK surgery in low- to moderate-risk cases using tissue from the UK eye banks and operated at our centre resulted in graft survival and endothelial cell counts over the first 2 years that were comparable to outcomes from international centres. DMEK endothelial cell counts in the first 12 months were superior to DSEK endothelial cell counts over the same interval in similar cases at our centre. We encountered a very low rebubble rate of $2 \%$ for DMEK, which may be related to our technique of temporarily elevating intraocular pressure with intracameral air for $10 \mathrm{~min}$ intra-operatively to assist graft attachment.

Acknowledgements The authors acknowledge David Johnson, PhD, for assistance in the database management.

Funding The authors declare no financial or proprietary interest in the materials presented. No funding or financial support was provided for this work.

\section{Compliance with ethical standards}

Conflict of interest The authors declare that they have no conflict of interest.

\section{References}

1. Melles GRJ, Lander F, Rietveld FJR. Transplantation of Descemet's membrane carrying viable endothelium through a small scleral incision. Cornea. 2002;21:415-8.

2. Melles GRJ, Ong T, Ververs B, van der Wees J. Descemet membrane endothelial keratoplasty. Cornea. 2006;25:987-90.

3. Ham L, Dapena I, van Luijk C, van der Wees J, Melles GRJ. Descemet membrane endothelial keratoplasty (DMEK) for Fuchs endothelial dystrophy: review of the first 50 consecutive cases. Eye (Lond). 2009;23:1990-8.

4. Deng SX, Lee WB, Hammersmith KM, Kuo AN, Li JY, Shen JF, et al. Descemet membrane endothelial keratoplasty: safety and outcomes. Ophthalmology. 2017. https://doi.org/10.1016/j.ophtha. 2017.08.015.

5. Oellerich S, Baydoun L, Peraza-Nieves J, Ilyas A, Frank L, Binder PS, et al. Multicenter study of 6-month clinical outcomes after Descemet's membrane endothelial keratoplasty. Cornea. 2017;36:1467-76.

6. Melles GRJ, Wijdh RHJ, Nieuwendaal CP. A technique to excise the Descemet membrane from a recipient cornea (descemetorhexis). Cornea. 2004;23:286-8.

7. Armitage WJ, Dick A, Bourne WM. Predicting endothelial cell loss and long-term corneal graft survival. Invest Ophthalmol Vis Sci. 2003;44:3326-31.

8. Fajgenbaum MA, Hollick EJ. Modeling endothelial cell loss after Descemet stripping endothelial keratoplasty. Cornea. 2017;36:553-60.

9. Price MO, Fairchild KM, Price DA, Price FW. Descemet's stripping endothelial keratoplasty: five-year graft survival and endothelial cell loss. Ophthalmology. 2011;118:725-9.

10. Wacker K, Baratz KH, Maguire LJ, McLaren JW, Patel SV. Descemet stripping endothelial keratoplasty for Fuchs' endothelial corneal dystrophy: five-year results of a prospective study. Ophthalmology. 2016;123:154-60.

11. Ang M, Soh Y, Htoon HM, Mehta JS, Tan D. Five-year graft survival comparing Descemet stripping automated endothelial keratoplasty and penetrating keratoplasty. Ophthalmology. 2016;123:1646-52.

12. Fajgenbaum M, Hollick EJ. Center and surgeon effect on outcomes of endothelial keratoplasty versus penetrating keratoplasty in the United Kingdom. Am J Ophthalmol. 2015;160:392-3.

13. Riddlesworth TD, Kollman C, Lass JH, Patel SV, Stulting RD, Benetz BA, et al. A mathematical model to predict endothelial cell density following penetrating keratoplasty with selective dropout from graft failure. Invest Ophthalmol Vis Sci. 2014; 55:8409-15.

14. Brissette A, Conlon R, Teichman JC, Yeung S, Ziai S, Baig K. Evaluation of a new technique for preparation of endothelial grafts for Descemet membrane endothelial keratoplasty. Cornea. 2015;34:557-9.

15. Dapena I, Moutsouris K, Droutsas K, Ham L, van Dijk K, Melles GRJ. Standardized "no-touch" technique for Descemet membrane endothelial keratoplasty. Arch Ophthalmol. 2011;129:88-94.

16. Rodríguez-Calvo-De-Mora M, Quilendrino R, Ham L, Liarakos VS, van Dijk K, Baydoun L, et al. Clinical outcome of 500 consecutive cases undergoing Descemet's membrane endothelial keratoplasty. Ophthalmology. 2015;122:464-70. 
17. Hamzaoglu EC, Straiko MD, Mayko ZM, Sáles CS, Terry MA. The first 100 eyes of standardized Descemet stripping automated endothelial keratoplasty versus standardized Descemet membrane endothelial keratoplasty. Ophthalmology. 2015;122:2193-9.

18. Deng SX, Sanchez PJ, Chen L. Clinical outcomes of Descemet membrane endothelial keratoplasty using eye bank-prepared tissues. Am J Ophthalmol. 2015;159:590-6.

19. Guerra FP, Anshu A, Price MO, Giebel AW, Price FW. Descemet's membrane endothelial keratoplasty: prospective study of 1year visual outcomes, graft survival, and endothelial cell loss. Ophthalmology. 2011;118:2368-73.

20. Siggel R, Adler W, Stanzel TP, Cursiefen C, Heindl LM. Bilateral Descemet membrane endothelial keratoplasty. Cornea. 2016;35:772-7.

21. Dapena I, Ham L, Droutsas K, Van Dijk K, Moutsouris K, Melles GR. Learning curve in Descemet's membrane endothelial keratoplasty: first series of 135 consecutive cases. Ophthalmology. 2011;118:2147-54.

22. Singh A, Zarei-Ghanavati M, Avadhanam V, Liu C. Systematic review and meta-analysis of clinical outcomes of Descemet membrane endothelial keratoplasty versus Descemet stripping endothelial keratoplasty/Descemet stripping automated endothelial keratoplasty. Cornea. 2017;36:1437-43.

23. Khor W-B, Han S-B, Mehta JS, Tan DTH. Descemet stripping automated endothelial keratoplasty with a donor insertion device: clinical results and complications in 100 eyes. Am J Ophthalmol. 2013;156:773-9.

24. Busin M, Bhatt PR, Scorcia V. A modified technique for Descemet membrane stripping automated endothelial keratoplasty to minimize endothelial cell loss. Arch Ophthalmol. 2008;126:1133.

25. Feng MT, Burkhart ZN, Price FW, Price MO. Effect of donor preparation-to-use times on Descemet membrane endothelial keratoplasty outcomes. Cornea. 2013;32:1080-2.

26. Ham L, Dapena I, Liarakos VS, Baydoun L, van Dijk K, Ilyas A, et al. Midterm results of Descemet membrane endothelial keratoplasty: 4 to 7 years clinical outcome. Am J Ophthalmol. 2016;171:113-21.

27. Schlögl A, Tourtas T, Kruse FE, Weller JM. Long-term clinical outcome after Descemet membrane endothelial keratoplasty. Am J Ophthalmol. 2016;169:218-26. 